\title{
Sjögren's Syndrome Associated with Liver and Neurological Disorders, and Malignant Lymphoma
}

Key words: hepatitis $\mathrm{C}$ virus, primary biliary cirrhosis, transverse myelitis, mucosa-associated lymphoid tissue

The 53-year-old woman reported in this issue (1), raises five interesting points: namely; Sjögren's syndrome (SS), primary biliary cirrhosis, hepatitis $\mathrm{C}$ virus (HCV) infection, transverse myelitis, and malignant lymphoma. These problems occurred in a single patient in a fairly short period from 1994 to 1998. How do these disorders relate to each other? Which one is upstream and which one is downstream?

See also $\mathrm{p} 260$.

SS is a chronic organ-specific autoimmune disease characterized by lymphocytic infiltration of the salivary and lacrimal glands, however, half of the SS patients develop systemic disorders associated with many kinds of autoantibodies. Systemic disorders include musculoskeletal, pulmonary, gastrointestinal, hematological, vascular, dermatologic, renal and nervous system disorders. In addition, about $5 \%$ of SS patients develop malignant lymphoma.

I would like to consider that the $\mathrm{HCV}$ infection was the first of the complicated disorders to start in this 53-year-old woman and that malignant lymphoma was the last event, and that SS was in the middle stream and primary biliary cirrhosis and transverse myelitis were related to SS.

The genetic abnormality of a suppressive mechanism for virus activation and an abnormal reaction to the virus is considerable to underlay in this patient. A concordance rate of $25 \%$ for the most common autoimmune diseases is shown in monozygotic twins, indicating genetic factors are the first to be considered. However, the fact that a 53-year-old woman is the target of SS and other autoimmune disorders as well as malignant lymphoma shows the importance of environmental factors.

HCV has a wide pathogenic potential that is not limited to diseases of the liver. HCV is a hepatotropic and lymphotropic virus and is found in epithelial cells of the parotid gland. HCV infection is associated with production of autoantibodies. Up to $30 \%$ of HCV patients develop SS and infection of HCV may be directly responsible for the clonal expansion of $\mathrm{B}$ cells (2).

SS is one of the common diseases associated with primary biliary cirrhosis which is an autoimmune chronic cholestatic liver disease characterized by the presence of anti-mitochondrial antibodies. T cell clones specific for pyruvate dehydrogenase complex (PDC)-E2 peptides were established from patients (3) and PDC-specific T cells are circulating in the peripheral blood of patients. Inappropriate expression of MHC class II molecules and PDC-2 antigens on biliary epithelial cells may stimulate $\mathrm{T}$ cells, leading to the chronic autoimmune reaction. Although PDC is a ubiquitous antigen, the specific expression of mitochondrial antigens in addition to HLA DR molecules on epithelial cells of the bile duct may explain the occurrence of organ-specific disease, primary biliary cirrhosis, just like that of SS.

Although central nervous disorders are reported in up to $25 \%$ of SS patients, this complication is rare in Japan (4). Transverse myelitis is a rare disorder and is sometimes associated with optic neuropathy. In these patients, recent tools to make a diagnosis are MRI findings on T2-wighted images in the spinal cord. In SS, hypergammaglobulinemic purpura is a disorder in which a complex of $7 \mathrm{~S} \mathrm{IgG} \mathrm{or} \mathrm{IgA} \mathrm{rheumatoid} \mathrm{factor} \mathrm{is}$ an etiologic factor to causing vasculitis. The same kind of etiological mechanism may have caused vasculitis of the spinal cord in this patient, although direct lymphocytic infiltration of the central nervous system was reported. An active immunological phenomenon such as an infiltration with macrophages in nerves may cause neurological disorders (4).

As for malignant lymphoma in SS, a high incidence of B cell lymphoma as well as monoclonal gammopathy was reported by many clinicians including us. In SS low-grade B cell lymphomas are most frequently observed, including mantle cell lymphoma (MCL), follicular cell lymphoma (FCL) and mucosa-associated lymphoid tissue (MALT) lymphoma (5). However, in our series of 20 malignant lymphomas, 10 lymphomas were of the nodal type and two out of 10 were of the diffuse large-cell type. MALT lymphomas can transform into highgrade lymphomas after up to 18 years. Diffuse lymphomas can be a result of transformation from low-grade B cell lymphomas. The vast majority of MALT lymphomas originate in a setting of chronic inflammation triggered by chronic infection or autoimmune disorders such as helicobacter pylori gastritis, SS and Hashimoto's thyroiditis. MALT lymphomas in SS are considered to arise in the lymphoepithelial lesion in the antigen-driven manner (6). In SS aberrant expression of class II molecules in conjunction with autoantigens such as $\alpha$-fodrin and etc., and the expression of CD80/86 molecules on the surface of activated ductal cells of salivary glands may be the first 
step to initiate the local autoimmune reaction. Activated T cells transiently express surface CD40L molecules which are strong components to stimulate B cells through CD40 molecules on the B cells. Bcl-2 overexpression may occur during the T-B cell interaction without translocation of chromosomes 14 and 18 , resulting in inhibition of apoptosis of B cells. Recently, apoptosis inhibitor gene API2 and a novel 18q gene MLT were reported in the $\mathrm{t}(11 ; 18)(\mathrm{q} 21 ; \mathrm{q} 21)$ to represent the most frequent structural abnormality of MALT lymphomas (7). Longevity of the B cell life span may allow some oncogenes such as p53 to be mutated. This kind of complex multistep process explains the occurrence of B cell lymphomas in SS from the chronic autoimmune inflammation.

Susumu SugaI, MD Kanazawa Medical University 1-1 Daigaku, Uchinada, Kahoku-gun, Ishikawa 920-0293

\section{References}

1) Wakatsuki T, Miyata M, Shishido S, et al. Sjögren's syndrome with primary biliary cirrhosis, complicated by transverse myelitis and malignant lymphoma. Intern Med 39: 260-265, 2000.

2) Ferri C, Monti M, La Civita L, et al. Infection of peripheral blood mononuclear cells by hepatitis $\mathrm{C}$ virus in mixed cryogloulinemia. Blood $\mathbf{8 2}$ 3701-3704, 1993.

3) Shimoda S, Nakamura M, Ishibashi H, et al. HLA DRB4 0101-restricted immunodominant $\mathrm{T}$ cell autoepitope of pyruvate dehydrogenase complex in primary biliary cirrhosis: evidence of molecular mimicry in human autoimmune diseases. J Exp Med 181: 1835-1845, 1995.

4) Tajima Y, Mito Y, Owada Y, et al. Neurological manifestations of primary Sjögren's syndrome in Japanese patients. Intern Med 36: 690-693, 1997.

5) Anaya J-M, McGuff HS, Banks PM, et al. Clinicopathological factors relating malignant lymphoma with Sjögren's syndrome. Semin Arthritis Rheum 25: 337-346, 1996.

6) Sugai S. Autoimmune diseases and neoplasm-malignant lymphoma in Sjögren's syndrome as a model. Jpn J Intern Med 88: 2032-2039, 1999 (in Japanese).

7) Dierlamm J, Baens M, Wlodarska I, et al. The apoptosis inhibitor gene API2 and novel 18q gene, MLT, are recurrently rearranged in the $t(11 ; 8)$ (q21; q21) associated with mucosa-associated lymphoid tissue lymphomas. Blood 93: 3601-3609, 1999. 